\title{
The Capacity and Energy Efficiency of Wireless Ad Hoc Networks with Multi-Packet Reception
}

\author{
Zheng Wang \\ wzgold@soe.ucsc.edu
}

\author{
Hamid R. Sadjadpour ${ }^{\dagger}$ \\ hamid@soe.ucsc.edu
}

\author{
J.J. Garcia-Luna-Aceves ${ }^{\ddagger}$ \\ jj@soe.ucsc.edu
}

\author{
Department of Electrical Engineering ${ }^{\dagger}$ and Computer Engineering ${ }^{\ddagger}$ \\ University of California, Santa Cruz, 1156 High Street, Santa Cruz, CA 95064, USA \\ ¥ Palo Alto Research Center (PARC), 3333 Coyote Hill Road, Palo Alto, CA 94304, USA
}

\begin{abstract}
We address the cost incurred in increasing the transport capacity of wireless ad hoc networks over what can be attained when sources and destinations communicate over multi-hop paths and nodes can transmit or receive at most one packet at a time. We define the energy efficiency $\eta(n)$ as the bitmeters of information transferred in the network for each unit energy. We compute the energy efficiency of many different techniques aimed at increasing the capacity of wireless networks and show that, in order to achieve higher transport capacity, a lower energy efficiency must be attained. Using the physical model, we compute the throughput capacity of random wireless ad hoc networks in which nodes are endowed with multi-packet reception (MPR) capabilities. We show that $\lambda(n)=\Theta\left(\frac{(R(n))^{(1-2 / \alpha)}}{n^{1 / \alpha}}\right)$ bits per second constitutes a tight upper and lower bound for the throughput capacity of random wireless ad hoc networks, where $\alpha>2$ is the path loss parameter in the physical model, $n$ is the total number of nodes in the network, and $R(n)$ is the MPR receiver range. In doing so, we close the gap between the lower and upper bounds for the throughput capacity of wireless networks in the physical model. Compared to the original result derived for plain routing by Gupta and Kumar, MPR achieves a capacity gain of at least $\Theta\left((\log n)^{\frac{\alpha-2}{2 \alpha}}\right)$ when $R(n)=\Theta(\sqrt{\log n / n})$.
\end{abstract}

\section{Categories and Subject Descriptors}

C.2.1 [Network Architecture and Design Wireless Communication]: [Computer-Communication Network]

\section{General Terms}

Performance, Theory

Permission to make digital or hard copies of all or part of this work for personal or classroom use is granted without fee provided that copies are not made or distributed for profit or commercial advantage and that copies bear this notice and the full citation on the first page. To copy otherwise, to republish, to post on servers or to redistribute to lists, requires prior specific permission and/or a fee.

MobiHoc'08, May 26-30, 2008, Hong Kong SAR, China.

Copyright 2008 ACM 978-1-60558-083-9/08/05 ...\$5.00.

\section{Keywords}

Capacity, Throughput, Multi-Packet Reception, Wireless Ad Hoc Networks, Energy Efficiency

\section{INTRODUCTION}

The seminal work by Gupta and Kumar [8] on the scaling laws of wireless ad hoc networks show that forwarding information from sources to destinations over multihop paths in which each relay is able to transmit or receive at most one packet at a time is not scalable. As a result, there has been a growing interest in the study of the capacity of ad hoc wireless networks and methods that can be used to improve the order capacity of such networks.

In this paper, we focus on the cost incurred by approaches aimed at increasing the order capacity of a wireless ad hoc network subject to multiple unicast flows. Section 2 provides a survey of such schemes, which include: Taking advantage of the in-network storage available in mobile nodes [7], increasing the available bandwidth of the network as the number of nodes increases [13], and establishing various forms of cooperation among senders and receivers (e.g., [1,14]).

Multi-packet reception (MPR) is a cooperative approach that enables each receiver to decode multiple concurrent transmissions within its reception radius. Ghez et al. $[5,6]$ and Tong et al. [17] present the first model of MPR in a framework for many-to-one communication. In this context, multiple nodes cooperate to transmit their packets simultaneously to the same node using directional antennas, multiuser detection (MUD), or multiple input multiple output (MIMO) techniques $[15,20,21]$. The receiver node utilizes MUD and successive interference cancelation (SIC) to decode multiple packets [18]. Recently, we [4] have shown that the throughput capacity with MPR is tightly bounded by $\Theta(R(n))$ under the protocol model. This represents a minimum gain of $\Theta(\log n)$ compared to the capacity bounds obtained by Gupta and Kumar for point-to-point communication under the protocol model. However, this work does not address the physical model.

Another cooperative technique aimed at increasing the capacity of wireless networks is network coding (e.g., $[9,23])$. However, while work is ongoing trying to determine the capacity gains that can be attained with $\mathrm{NC}$ in multicast applications, Liu et al. [11] have shown that NC does not provide any order capacity increase for unicast applications, which is the focus of this paper.

The first contribution of this paper is to compute the 
throughput capacity of random wireless ad hoc networks under the physical model assumption when all nodes are endowed with MPR. Section 3 presents the network model we use to obtain the upper and lower bounds on the throughput capacity of wireless networks with MPR, which are derived in Section 4. We show that $\Theta\left(\frac{(R(n))^{(1-2 / \alpha)}}{n^{1 / \alpha}}\right)$ bits per second constitutes a tight bound for the throughput capacity per node in random wireless ad hoc networks, where $R(n)$ and $\alpha$ are the MPR receiver range and channel path loss parameter, respectively. When $R(n)=\Theta\left(\sqrt{\frac{\log n}{n}}\right)$, the throughput capacity is tight bounded by $\Theta\left(\frac{(\log n)^{\frac{1}{2}-\frac{1}{\alpha}}}{\sqrt{n}}\right)$. This is a gain of $\Theta\left((\log n)^{\frac{\alpha-2}{2 \alpha}}\right)$ compared to the bound $\Theta(1 / \sqrt{n})$ in [8] and [3]. The assumptions we use to obtain these results are similar to those made by Gupta and Kumar [8], except that each node is equipped with MPR capabilities.

Gupta and Kumar showed that, under the physical model, the throughput capacity of a wireless network has lower and upper bounds of $\Theta(\sqrt{1 / n \log n})$ and $\Theta(\sqrt{1 / n})$, respectively [8]. Subsequently, Franceschetti et al. [3] closed the gap between these two bounds and obtained an upper bound of $\Theta(\sqrt{1 / n})$ under the physical model using percolation theory. The second contribution of this paper consists of further closing the gap between the upper and lower bounds on the throughput capacity of wireless networks with MPR under the physical model.

Intuitively, there must be a price paid in all the approaches proposed to date that increase the capacity of wireless networks, including MPR of course. This price is the energy required to transport information across the network. Many wireless ad hoc and sensor networks have limited power and energy for each node. Hence, capacity alone is not necessarily the only performance metric against which the effectiveness of a given approach should be evaluated. Energy and power consumption efficiency is also an important metric that must be considered. The third contribution of this paper is the introduction of a new metric to evaluate the performance of wireless ad hoc networks in terms of energy efficiency. Section 5 introduces a new parameter to quantify how many bit-meters of information are transferred across the network per each unit energy. We call this metric energy efficiency, computed by normalizing the transport capacity by the total transmitted power and measured in units of bitmeters per joule. We compute the energy efficiency of many existing techniques $[3,8,14]$ and compare them to the energy efficiency of MPR approach. We show that MPR provides a tradeoff between throughput capacity, node decoding complexity, and energy efficiency in random wireless ad hoc networks. We also show that in all existing techniques $[3,8,14]$ including MPR, achieving higher throughput capacity leads to a lower energy efficiency. Note that the transport capacity for random wireless ad hoc networks is defined in bits per second while for arbitrary wireless ad hoc networks, the transport capacity is measured in bit-meters per second [8]. The focus of this paper is only on random wireless ad hoc networks.

Previous work $[16,19]$ has suggested the concept of bits per joule capacity to evaluate how much information can be transmitted with each unit energy. Our definition of energy efficiency is an extension of this prior work for transport capacity in wireless ad hoc networks. Section 6 discusses several possible implications of this study.

\section{RELATED WORK}

Many contributions have been made on the capacity of wireless networks subject to unicast traffic. Under the physical model assumption, Gupta and Kumar [8] showed that the throughput capacity of random wireless ad hoc networks has lower and upper bounds of $\Theta(\sqrt{1 / n \log n})$ and $\Theta(\sqrt{1 / n})$, respectively. Franceschetti et al. [3] closed the gap between these two bounds and obtained a tight bound of $\Theta(\sqrt{1 / n})$ using percolation theory. In this approach, all communication is simple point-to-point without any cooperation between senders and receivers. Communication between relays is kept at short distances with multi-hop transmissions in backbone paths, while nodes require to transmit longer distances to reach the backbone paths.

Several techniques have been proposed to improve the capacity of wireless networks. Grossglauser and Tse [7] demonstrated that a non-vanishing capacity can be attained at the price of long delivery latencies by taking advantage of longterm storage in mobile nodes. Negi and Rajeswaran [13] showed that, by changing the physical layer assumptions, we can increase the capacity of wireless networks. Allowing bandwidth expansion using ultra wide-band (UWB) technology, throughput capacity $O\left((n \log n)^{(\alpha-1) / 2}\right)$ and

$\Omega\left(\frac{n^{(\alpha-1) / 2}}{(\log n)^{(\alpha+1) / 2}}\right)$ can be attained as the upper and lower bounds, respectively at the expense of allowing the bandwidth to increase as a function of the number of nodes in the network. Zhang and Hou [22] closed the gap in [13] by applying percolation theory [3]. We can also increase the throughput capacity by using multiple channels [10] or sender-receiver cooperation [1]. Recently, Ozgur et al. [14] demonstrated that the capacity of random wireless ad hoc network scales linearly with $n$ by allowing nodes to cooperate intelligently using distributed MIMO communications.

Throughput capacity is only one figure of merit in evaluating different techniques. There are also other factors such as delay, energy consumption, storage, and other factors that should be evaluated. Therefore, there is always a tradeoff between throughput capacity and other factors in the network. El Gamal et al. [2] characterized the fundamental throughput-delay tradeoff for both static and mobile networks.

\section{NETWORK MODEL}

We consider a dense wireless ad hoc network with $n$ nodes distributed uniformly in a square of unit area. Hence, in our model, as $n$ goes to infinity, the density of the network also goes to infinity. Our capacity analysis is based on the extension of physical model for dense networks introduced by Gupta and Kumar [8].

Definition 3.1. Physical Model with Plain Routing In the physical model of dense random wireless ad hoc networks [8], a successful communication occurs if signal to interference and noise ratio (SINR) of the pair of transmitter $i$ and receiver $j$ satisfies

$$
\operatorname{SINR}_{i \rightarrow j}=\frac{P g_{i j}}{B N_{0}+\sum_{k \neq i, k=1}^{n} P g_{k j}} \geq \beta,
$$


where $P$ is the transmit power of a node, $g_{i j}$ is the channel attenuation factor between nodes $i$ and $j$, and $B N_{0}$ is the total noise power. The channel attenuation factors $g_{i j}$ and $g_{k j}$ are only functions of the distance under the simple path loss propagation model we assume [8]. Therefore, $g_{i j}=\left|X_{i}-X_{j}\right|^{-\alpha}$ in which $\alpha>2$ is the path-loss parameter and $X_{i}$ represents the Cartesian location of node $i$.

However, in the physical model of MPR, each receiving node has a receiver range such that all the nodes transmitting within this range will be decoded by the receiver. Consequently, the definition of physical model should incorporate this fact in order to better represent this new manyto-one communication scheme. The following proposition states the decoding procedure for MPR using SIC. Note that, with MPR, we can either decode the received signal for multiple transmitters jointly using maximum likelihood decoding or decode transmitters sequentially as long as the $S I N R$ condition is satisfied. Definition 3.3 below describes the condition that satisfies the minimum required SINR.

Proposition 3.2. The transmitter-receiver pair with maximum SINR is the nearest set of transmitters, after decoding and subtracting this group from the received signal, the set with the next highest SINR is the second nearest group of transmitters, and this continues; i.e., receivers decode the information from the nearest transmitters to farthest ones whose positions are the maximum distance inside of communication range.

Because the channel propagation model is based on the path-loss parameter, it is clear from (1) that the node (or group of nodes) with the closest distance to the receiver has the highest SINR. After decoding this (their) packet(s) and subtracting it (them) from the received data, it is obvious that the next packet(s) with highest SINR is (are) from the second closest node(s) to the receiver node and this procedure can continue. At a given time $t$, the decoding procedure for any receiver $j$ in the MPR scheme is sequential, i.e., a receiver decodes the information from the highest SINR to the lowest SINR for MPR with SIC.

Essentially, this proposition states that each group of transmissions from some transmitters can be decoded if and only if the previous group of transmissions from transmitters that are closer to the receiver node was decoded first by the receiver node. The last decoded node occurs at the edge of the circle whose radius is $R(n)$.

Definition 3.3. Physical Model with Multi-packet Reception

In the physical model of dense random wireless ad hoc networks [8], the transmissions from all of the transmitters centered around a receiver $j$ with a distance smaller or equal to $R(n)$ occur successfully if the SINR of the transmitter $Z(R(n))$ at the edge of this receiver circle satisfies

$$
\mathrm{SINR}_{Z(R(n)) \rightarrow j}=\frac{P g_{Z(R(n)) j}}{B N_{0}+\sum_{k \notin A_{Z(R(n))}} P g_{k j}} \geq \beta,
$$

where $g_{Z(R(n)) j}$ is the channel attenuation factor between nodes $Z(R(n))$ and $j$ and $B N_{0}$ is the total noise power. $A=\pi R^{2}(n)$ is the receiver communication range (circle) centered around the receiver $j$. The channel attenuation factors $g_{Z(R(n)) j}$ and $g_{k j}$ are only functions of the distance, i.e., $g_{Z(R(n)) j}=\left|X_{Z(R(n))}-X_{j}\right|^{-\alpha}$ and $g_{k j}=\left|X_{k}-X_{j}\right|^{-\alpha}$.
Any transmission outside the receiver range is considered interference while all the transmissions inside receiver range will be decoded jointly or separately. For this reason, we denote the interference inside area $A$ as constructive interference, because it consists of transmissions that will be eventually decoded, while all the transmissions from nodes outside of area $A$ are called destructive interference and are not decoded. Note that for the MPR model, the receiver range $R(n)$ defines the area where the receiver is capable of decoding, which contrasts with point-to-point communication [8], for which the transmission range $r(n)$ defines the possible area where the receiver can decode, given that only one transmission is successful at a receiver. Given that any transmitter that is closer to the receiver has a smaller channel attenuation compared to the edges of the circle, it is easy to show that the SINR of these transmitter nodes are always greater than the value in Equation (2) if these nodes are decoded jointly or separately depending on the distribution of these nodes around the receiver node $j$.

We assume that nodes cannot transmit and receive at the same time, which means half-duplex communication. The capacity between transmit node $i$ and receive node $j$ is defined as $C_{i j}=B \log (1+S I N R)$ bits/sec. In the work by Gupta and Kumar [8], $C_{i j}$ can be a constant value $W$ if and only if $S I N R$ is guaranteed to be larger than a constant $\beta$. We follow a similar assumption in this paper. We present the following definition from Gupta and Kumar's work [8] for completeness of the presentation.

Definition 3.4. Feasible throughput capacity of unicast: " A throughput of $\lambda(n)$ bits per second for each node is feasible if there is a spatial and temporal scheme for scheduling transmissions, such that by operating the network in a multihop fashion and buffering at intermediate nodes when awaiting transmission, every node can send $\lambda(n)$ bits per second on average to its chosen destination nodes. That is, there is $a T<\infty$ such that in every time interval $[(i-1) T, i T]$ every node can send $T \lambda(n)$ bits to its corresponding destination node."

Definition 3.5. Order of throughput capacity: $\lambda(n)$ is said to be of order $\Theta(f(n))$ bits per second if there exist deterministic positive constants $c$ and $c^{\prime}$ such that

$$
\left\{\begin{array}{l}
\lim _{n \rightarrow \infty} \operatorname{Prob}(\lambda(n)=c f(n) \text { is feasible })=1 \\
\lim _{n \rightarrow \infty} \operatorname{Prob}\left(\lambda(n)=c^{\prime} f(n) \text { is feasible }\right)<1 .
\end{array}\right.
$$

The distribution of nodes in random networks is uniform. Therefore, if there are $n$ nodes in a unit square, then the density of nodes equals $n$. Hence, if $|S|$ denotes the area of space region $\mathrm{S}$, the expected number of the nodes, $E\left(N_{S}\right)$, in this area is given by $E\left(N_{S}\right)=n|S|$. Let $N_{j}$ be a random variable defining the number of nodes in $S_{j}$. Then, for the family of variables $N_{j}$, we have the following standard results known as the Chernoff bound [12]:

LEMma 3.6. Chernoff bound

$$
\begin{aligned}
& \text { - For any } \delta>0, P\left[N_{j}>(1+\delta) n\left|S_{j}\right|\right]<\left(\frac{e^{\delta}}{(1+\delta)^{1+\delta}}\right)^{n\left|S_{j}\right|} \\
& \text { - For any } 0<\delta<1, P\left[N_{j}<(1-\delta) n\left|S_{j}\right|\right]<e^{-\frac{1}{2} n\left|S_{j}\right| \delta^{2}}
\end{aligned}
$$

Combining these two inequalities we have, for any $0<\delta<1$ :

$$
P\left[\left|N_{j}-n\right| S_{j}||>\delta n\left|S_{j}\right|\right]<e^{-\theta n\left|S_{j}\right|}
$$


where $\theta=(1+\delta) \ln (1+\delta)-\delta$ in the case of the first bound, and $\theta=\frac{1}{2} \delta^{2}$ in the case of the second bound.

Therefore, for any $\theta>0$, there exist constants such that deviations from the mean by more than these constants occur with probability approaching zero as $n \rightarrow \infty$. An event occurs with high probability (w.h.p.) if its probability tends to one as $n \rightarrow \infty$. It follows that, w.h.p., we can get a very sharp concentration on the number of nodes in an area, so we can find the achievable lower bound w.h.p., provided that the upper bound (mean) is given. In the next section, we first derive the upper bound, and then use the Chernoff bound to prove the achievable lower bound w.h.p..

\section{THROUGHPUT CAPACITY WITH MPR}

To compute the upper and lower bounds, we first describe some definitions and preliminary results from our earlier work [4].

The per-node throughput capacity of the network is defined as the number of bits per second that every node can transmit w.h.p. to its destination. Note that throughput capacity is equivalent to transport capacity in this paper. Transport capacity is defined in units of bits per second in random networks and bit-meters per second in arbitrary networks as originally described in [8]. In random networks, source-destination distance is on average the same order for all pairs and therefore, the distance is simply a normalization factor. Since this paper only discusses random networks, we use bits per second unit for transport capacity consistent with the definition in [8].

A cut $\Gamma$ is a partition of the vertices (i.e. nodes in the wireless networks) of a graph into two sets. The cut capacity is defined to be the sum of bandwidth of all the edges crossing the cut that can transmit simultaneously. Min-cut is a cut whose capacity is the minimum value among the capacity of all cuts. For the wireless networks, we use the concept of sparsity cut, as defined by Liu et al. [11], instead of mincut, to take into account the differences between wired and wireless links.

In the 2-D case, the cut length $l_{\Gamma}$ is defined as the length of the cut line segment. For the square region illustrated in Fig. 1, the middle line induces a sparsity cut $\Gamma$. Because nodes are uniformly deployed in a random network, such a sparsity cut captures the traffic bottleneck of these random networks on average.

The sparsity-cut capacity is upper bounded by deriving the maximum number of simultaneous transmissions across the cut.

Let $R(n)$ be the radius of the receiver range $A$, i.e., $A=$ $\pi R^{2}(n)$. Given that we assume omni-antenna broadcasting, this is the radius that distinguishes the decode-able transmitter nodes from the interference.

We assume that each disk with radius $R(n)$ centered at any receiver is disjoint from the other disks centered at the other receivers. It will be shown later that this assumption is necessary in order to guarantee that the physical model condition, $S I N R \geq \beta$, is satisfied.

\subsection{Upper Bound}

LEMMA 4.1. The asymptotic throughput capacity of a sparsity cut $\Gamma$ for a unit square region has an upper bound of $\frac{\pi l_{\Gamma} n W}{2} \frac{R^{2}(n)}{D(n)}$, where, $R(n)$ and $D(n)$ are receiver range and

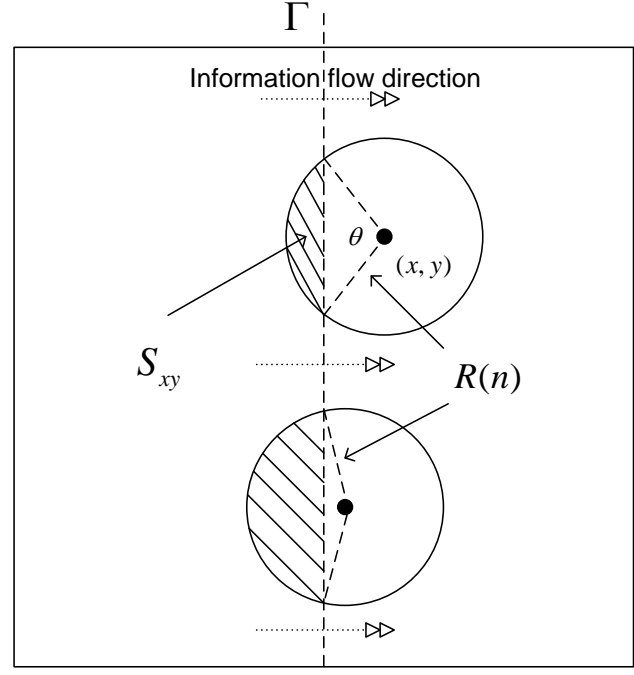

Figure 1: For a receiver at location $(x, y)$, all the nodes in the shaded region $S_{x y}$ can send a message successfully and simultaneously.

division range of MPR respectively. Fig. 2 illustrates $R(n)$ and $D(n)$.

Proof. The cut capacity is upper bounded by the maximum number of simultaneous transmissions across the cut. We observe from Fig. 1 that all the nodes located in the shaded area $S_{x y}$ can send their packets to the receiver node located at $(x, y)$. These nodes lie in the left side of the cut $\Gamma$ within an area called $S_{x y}$ and the assumption is that all these nodes are sending packets to the right side of the cut $\Gamma$.

For a node at location $(x, y)$, any node in the disk of radius $R(n)$ can transmit information to this receiver simultaneously and the node can successfully decode those packets. In order to obtain an upper bound, we only need to consider edges that cross the cut. Let us first consider all possible nodes in the $S_{x y}$ region that can transmit to the receiver node. By drawing a circle of radius $R(n)$ centered at $(x, y)$, this region is illustrated in Fig. 1 as $S_{x y}$. Because nodes are uniformly distributed, the average number of transmitters located in $S_{x y}$ is $n \times S_{x y}$. The number of nodes that are able to transmit at the same time from left to right is upper bounded as a function of $S_{x y}$.

The area of $S_{x y}$ is

$$
S_{x y}=\frac{1}{2} R^{2}(n)(\theta-\sin \theta) .
$$

This area is maximized when $\theta=\pi$,

$$
\max _{0 \leq \theta \leq \pi}\left[S_{x y}\right]=\frac{1}{2} \pi R^{2}(n)
$$

We can compute the total information capacity $C_{j}$ for one receiver $j$ at the right side of the cut as

$$
C_{j}=\frac{1}{2} \pi n W R^{2}(n) .
$$

The constraint to guarantee that Eq. (7) is true for all of the nodes inside the circle of radius $R(n)$, is to satisfy $S I N R_{i \in \mathrm{S}_{x y}} \geq \beta$. For this reason, the circles whose nodes are transmitting concurrently must be away from each other far 


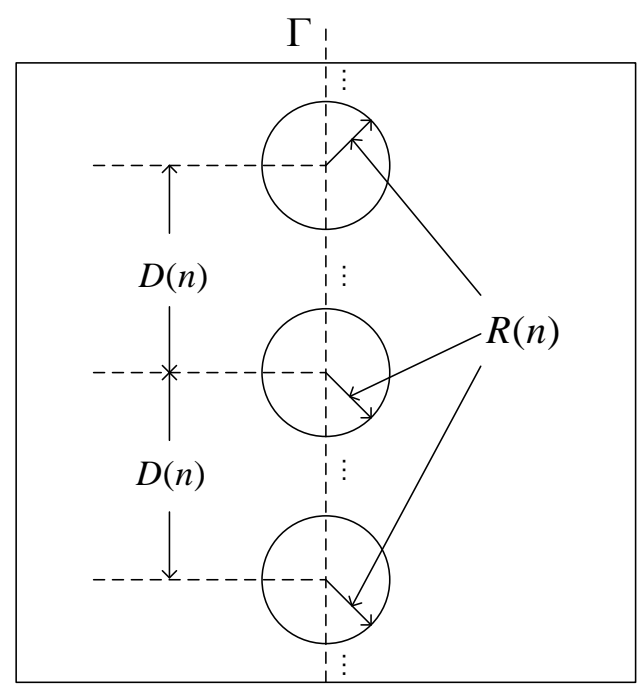

Figure 2: Upper bound design of the network

enough $(D(n) \geq 2 R(n))$ as shown in Fig. 2. Therefore, the total throughput capacity $C(n)$ across the sparsity cut is

$$
C(n) \leq\left(\left\lfloor\frac{l_{\Gamma}}{D(n)}\right\rfloor+1\right) C_{j}<\frac{\pi n W R^{2}(n)\left(l_{\Gamma}+D(n)\right)}{2 D(n)} .
$$

Note that $D(n)$ and $R(n)$ are decreasing functions of $n$, and $\lim \left(l_{\Gamma}+D(n)\right)=l_{\Gamma}$ asymptotically because $\lim D(n)=$ 0 as $n \rightarrow \infty$. This proves the lemma.

LEMMA 4.2. The per source-destination throughput of MPR scheme in a 2-D random network is upper bounded by $O\left(\frac{R^{2}(n)}{D(n)}\right)$.

Proof. From lemma 4.1, there are $l_{\Gamma} / D(n)$ different circles of radius $R(n)$ each of them having $\Theta\left(n R^{2}(n)\right)$ nodes on average. Therefore, the average per node throughput capacity can be derived as

$$
\lambda(n)=\frac{C(n)}{n}=O\left(\frac{R^{2}(n)}{D(n)}\right) .
$$

To derive an upper bound for the throughput capacity, we need to obtain a minimum $D(n)$, such that it guarantees $S I N R_{Z(R(n))} \geq \beta$. From Proposition 3.2, the decoding sequence of transmissions is from nearest nodes to farthest nodes, i.e., the information of the next transmitter in the communication range can be decoded if and only if the previous one is decoded successfully and then it is subtracted from the received data. So if the SINR of the outmost node can be decoded, then all of the nodes inside that circle can be decoded separately or at least jointly. Based on this assumption, we only need to compute the SINR of the farthest nodes $Z(R(n))$ (i.e., at the conjunction edge of the communication circle) to make sure $\operatorname{SINR}_{Z(R(n))} \geq \beta$. Therefore, the upper bound capacity exists if maximizing this capacity is equivalent of maximizing the following function.

$$
\max _{\operatorname{SINR}_{Z(R(n))} \geq \beta} \lambda(n)=\max _{\operatorname{SINR}_{Z(R(n))} \geq \beta} \frac{R^{2}(n)}{D(n)}
$$

Note that the throughput capacity is maximized by minimizing $D(n)$, while if this value is too small, then Eq. (2) will not be satisfied. Our aim is to find the optimum value for $D(n)$ such that both conditions are satisfied. The following theorem establishes the optimum value that will satisfy Eq. (2).

THEOREM 4.3. The per source-destination throughput of $M P R$ scheme in a 2-D random network is upper bounded by $O\left(\frac{(R(n))^{(1-2 / \alpha)}}{n^{1 / \alpha}}\right)$.

PROOF. In order to compute the upper bound, we derive the $S I N R$ for the node that is in a circle close to the edge of the network. For this receiver node, the Euclidean distances of interfering nodes are at $(i D(n)+R(n))$ if we assume all interfering nodes are at the farthest distance from the receiver node. Then the SINR of the transmitter node that is located at the circumference of the communication circle is given by

$$
\begin{aligned}
\operatorname{SINR}_{Z(R(n))} & \leq \frac{P / R^{\alpha}(n)}{\frac{\pi}{2} n R^{2}(n) \sum_{i=1}^{l_{\Gamma} / D(n)} \frac{P}{(i D(n)+R(n))^{\alpha}}} \\
& \leq\left(\frac{D(n)}{R(n)}\right)^{\alpha} \frac{1}{\frac{\pi}{2} n R^{2}(n) \sum_{i=1}^{l_{\Gamma} / D(n)} \frac{1}{\left(i+\frac{1}{2}\right)^{\alpha}}}
\end{aligned}
$$

The second inequality above stems from the fact that $\frac{R(n)}{D(n)} \leq$ $\frac{1}{2}$. Note that $l_{\Gamma} / D(n)$ approaches infinity when $n \rightarrow \infty$; therefore, the summation $\sum_{i=1}^{l_{\Gamma} / D(n)} \frac{1}{\left(i+\frac{1}{2}\right)^{\alpha}}$ converges to a bounded value. This means that there are constant values $c_{3}$ and $c_{4}$ such that

$$
c_{3} \leq \sum_{i=1}^{l_{\Gamma} / D(n)} \frac{1}{\left(i+\frac{1}{2}\right)^{\alpha}} \leq \sum_{i=1}^{l_{\Gamma} / D(n)} \frac{1}{(i)^{\alpha}} \leq c_{4} .
$$

Combining (11) and (1), the SINR constraint can be revised as

$$
\beta \leq \operatorname{SINR}_{Z(R(n))} \leq\left(\frac{D(n)}{R(n)}\right)^{\alpha} \frac{2}{\pi c_{3} n R^{2}(n)} .
$$

Then the relationship between $R(n)$ and $D(n)$ can be expressed as

$$
D(n) \geq\left(\frac{c_{3} \beta \pi}{2}\right)^{\frac{1}{\alpha}} n^{\frac{1}{\alpha}}(R(n))^{(1+2 / \alpha)} .
$$

From Eqs. (9) and (14), the upper bound of the throughput capacity is computed as

$$
\lambda(n)=O\left(\frac{R^{2}(n)}{D(n)}\right)=O\left(\frac{(R(n))^{(1-2 / \alpha)}}{n^{1 / \alpha}}\right) .
$$

The above upper bound is derived based on the assumption that the SINR for the nodes that are located on the circumference of communication circle $A$ of radius $R(n)$ satisfy the physical model, i.e., $\operatorname{SINR}_{Z(R(n))} \geq \beta$. Note that successive decoding of nodes in $A$ starts with nodes with the highest $S I N R$ or equivalently with the closest distance to the receiver $j$. Let's define $U_{i} \in A$ as a subset of the set $A$ that contains a group of nodes in the communication circle with the closest distance to the receiver $j$ that will be decoded jointly in the next step. Then it is easy to show that $S I N R_{U_{i}} \geq S I N R_{Z(R(n))} \geq \beta$. We will prove this in the 
next section and show that this upper bound capacity is also an achievable lower bound.

\subsection{Lower Bound}

Before proving the lower bound, we first compute the number of nodes that transmit simultaneously from each communication circle.

We have derived the upper bound in the previous section and then the Chernoff Bound is used to prove the achievable lower bound w.h.p.

Next we prove that, when $n$ nodes are distributed uniformly over a square area, we have simultaneously at least $\frac{l_{\Gamma}}{D(n)}$ circular regions (see fig. 1), each one containing

$\Theta\left(n R^{2}(n)\right)$ nodes w.h.p.. The objective is to find the achievable lower bound using the Chernoff bound, such that the distribution of the number of edges across the cut is sharply concentrated around its mean, and hence in a randomly chosen network, the actual number of edges crossing the sparsity cut is indeed $\Theta\left(\frac{(R(n))^{(1-2 / \alpha)}}{n^{(1 / \alpha)}}\right)$ w.h.p..

THEOREM 4.4. Each area $A_{j}$ with circular shape of radius $R(n)$ contains $\Theta\left(n R^{2}(n)\right)$ nodes w.h.p. and uniformly for all values of $j, 1 \leq j \leq \frac{l_{\Gamma}}{D(n)}$ under the condition that $R(n) \geq$ $\Theta\left(\sqrt{\frac{\log n}{n}}\right)$. Equivalently, this can be expressed as

$$
\lim _{n \rightarrow \infty} P\left[\bigcap_{j=1}^{l_{\Gamma} / D(n)}\left|N_{j}-E\left(N_{j}\right)\right|<\delta E\left(N_{j}\right)\right]=1,
$$

where $\delta$ is a positive arbitrarily small value close to zero.

Proof. From Equation (4), for any given $0<\delta<1$, there exists a $\theta>0$ such that

$$
P\left[N_{j}-\left|E\left(N_{j}\right)\right|>\delta E\left(N_{j}\right)\right]<e^{-\theta E\left(N_{j}\right)}=e^{-\theta n\left|A_{j}\right|} .
$$

Thus, we can conclude that the probability that the value of the random variable $N_{j}$ deviates by an arbitrarily small constant value from the mean tends to zero as $n \rightarrow \infty$. This is a key step in showing that when all the events $\bigcap_{j=1}^{l_{\Gamma} / D(n)} \mid N_{j}$ $E\left(N_{j}\right) \mid<\delta E\left(N_{j}\right)$ occur simultaneously, then all $N_{j}$ s converge uniformly to their expected values. Utilizing the union bound, we arrive at

$$
\begin{aligned}
& P\left[\bigcap_{j=1}^{l_{\Gamma} / D(n)}\left|N_{j}-E\left(N_{j}\right)\right|<\delta E\left(N_{j}\right)\right] \\
= & 1-P\left[\bigcup_{j=1}^{l_{\Gamma} / D(n)}\left|N_{j}-E\left(N_{j}\right)\right|>\delta E\left(N_{j}\right)\right] \\
\geq & 1-\sum_{j=1}^{l_{\Gamma} / D(n)} P\left[\left|N_{j}-E\left(N_{j}\right)\right|>\delta E\left(N_{j}\right)\right] \\
> & 1-\frac{l_{\Gamma}}{D(n)} e^{-\theta E\left(N_{j}\right)} .
\end{aligned}
$$

Because $E\left(N_{j}\right)=\frac{\pi}{2} n R^{2}(n)$ (see (6)), the final result is

$$
\begin{aligned}
& \lim _{n \rightarrow \infty} P\left[\bigcap_{j=1}^{l_{\Gamma} / D(n)}\left|N_{j}-E\left(N_{j}\right)\right|<\delta E\left(N_{j}\right)\right] \\
& \geq 1-\frac{l_{\Gamma}}{D(n)} e^{-\frac{\theta \pi n R^{2}(n)}{2}} \geq 1-\frac{l_{\Gamma}}{2 R(n)} e^{-\frac{\theta \pi n R^{2}(n)}{2}}
\end{aligned}
$$

If $R(n) \geq \sqrt{\frac{c_{5} \log n}{n}}=\Theta\left(\sqrt{\frac{\log n}{n}}\right)$ and as $n \rightarrow \infty$, then $\frac{e^{-\frac{\theta \pi n R^{2}(n)}{2}}}{R(n)} \rightarrow 0$, when $\theta>1 / \pi c_{5}$. Here, the key constraint of $R(n)$ is given as

$$
R(n) \geq \Theta\left(\sqrt{\frac{\log n}{n}}\right)
$$

Eq. (20) is equivalent to the connectivity condition in the protocol model $[4,8]$. It is interesting to note that we did not really use connectivity criterion in the physical model, however, it turns out that the minimum distance for the receiver range in MPR model is equivalent to the connectivity constraint in random networks.

The above theorem demonstrates that w.h.p., there are indeed $\Theta\left(n R^{2}(n)\right)$ nodes in each communication region with the constraint in (20). The achievable capacity is only feasible when the receiver range of each node in MPR scheme is at least equal to the connectivity criterion of transmission range in point-to-point communication [8]. Combining the result of Eq. (15) in Theorem 4.3 and (20) in Theorem 4.4, we can state the following theorem for the lower bound of throughput capacity, which implies the lower bound order capacity achieves the upper bound.

THEOREM 4.5. The per source-destination throughput capacity of MPR scheme in a 2-D static wireless ad hoc network is lower bounded by $\Omega\left(\frac{(R(n))^{(1-2 / \alpha)}}{n^{1 / \alpha}}\right)$ provided that $R(n) \geq \Theta\left(\sqrt{\frac{\log n}{n}}\right)$, which means the tight bound is at least $\Theta\left(\frac{(\log n)^{\frac{1}{2}-\frac{1}{\alpha}}}{\sqrt{n}}\right)$ for $\alpha>2$.

Proof. We first prove that Eq. (15) is an achievable bound and then by applying the minimum receiver range constraint in Eq. (20), we derive the lower bound for this theorem.

In order to derive the achievable lower bound, we design a scheme for separating decode-able transmitter nodes inside the communication circle and interference, such that $S I N R_{Z(R(n))} \geq \beta_{1}$. Similar to the derivations in Eq. (11) and using Fig. 2, it is clear that the SINR is minimized when the largest value for interference is considered. This value is achieved when we compute the interference for a receiver node in the middle of the network and use the closest possible distance to the receiver node ${ }^{1}$. This lower bound can be written as

$$
\operatorname{SINR}_{Z(R(n))} \geq \frac{\frac{P}{R^{\alpha}(n)}}{B N_{0}+\frac{\pi}{2} n R^{2}(n) \sum_{i=1}^{l_{\Gamma} / 2 D(n)} \frac{2 P}{(i D(n)-R(n))^{\alpha}}} .
$$

Assume that $D(n)$ satisfies the condition in Eq. (14). If we use the constraint for $R(n)$ in (20), we arrive at

$$
\frac{D(n)}{R(n)} \geq\left(\frac{c_{3} \beta \pi}{2}\right)^{\frac{1}{\alpha}} n^{\frac{1}{\alpha}}(R(n))^{2 / \alpha} \geq \Theta\left((\log n)^{\frac{1}{\alpha}}\right),
$$

which illustrates that $R(n)$ can be ignored compared with $D(n)$ for large values of $n$, i.e., $n \rightarrow \infty$. We now evaluate

\footnotetext{
${ }^{1}$ Note that the difference between maximum and minimum value of interference is a constant value
} 
the asymptotic behavior of (21) when $n \rightarrow \infty$. Combining Eqs. (22) and (21), $S I N R_{Z(R(n))}$ can be lower bounded by

$$
\begin{aligned}
\lim _{n \rightarrow \infty} \operatorname{SINR}_{Z(R(n))} & \geq\left(\frac{D(n)}{R(n)}\right)^{\alpha} \frac{1}{\pi n R^{2}(n) \sum_{i=1}^{l_{\Gamma} / D(n)} \frac{1}{i^{\alpha}}} \\
& \geq\left(\frac{D(n)}{R(n)}\right)^{\alpha} \frac{1}{\pi c_{4} n R^{2}(n)} \geq \frac{c_{3}}{2 c_{4}} \beta=\beta_{1} .
\end{aligned}
$$

This inequality is derived using Eqs. (14) and (12), together with the fact that the second term in the denominator of $S I N R$ goes to infinity when $n \rightarrow \infty$ and, therefore, we can drop the first term related to the noise. Using the same arguments introduced for the computation of the upper bound, we can show that a non-zero value for $\operatorname{SINR}_{Z(R(n))}$ can be achieved which implies that the throughput capacity can be achieved asymptotically.

The above theorem demonstrates that a gain of at least $\Theta\left((\log n)^{\frac{\alpha-2}{2 \alpha}}\right)$ can be achieved compared with the results by Gupta and Kumar [8] and Franceschetti et al. [3]. Combining Theorems 4.3 and 4.5, we arrive at our first major contribution of this paper.

THEOREM 4.6. The per source-destination throughput capacity of MPR scheme in a 2-D static wireless ad hoc network is tight bounded as $\Theta\left(\frac{(R(n))^{(1-2 / \alpha)}}{n^{1 / \alpha}}\right)$. The minimum receiver range is lower bounded as $R(n) \geq \Theta\left(\sqrt{\frac{\log n}{n}}\right)$, which implies a lower tight bound of $\Theta\left(\frac{(\log n)^{\frac{1}{2}-\frac{1}{\alpha}}}{\sqrt{n}}\right)$.

Note that this result shows that we can close the gap in the physical model similar to the results derived by Franceschetti et al. [3] but achieving higher throughput capacity with MPR.

\section{ENERGY EFFICIENCY}

This section focuses on the energy efficiency of schemes aimed at increasing the throughput (transport) capacity of wireless ad hoc networks. Many wireless sensor and ad hoc networks are energy and power limited systems and it is natural to ask what the price of achieving higher capacities in wireless ad hoc networks is.

The transport capacity was originally defined in [8] based on bits per second for random networks and bit-meters per second for arbitrary networks. Recently, a new definition of bits-per Joule was defined [16] that takes into consideration the energy consumption that is required to transmit information bits.

In order to incorporate the effect of energy consumption for communication in wireless networks, we define bit-meters per Joule, or simply energy efficiency. This new metric is a measure for evaluating the energy efficiency of the transport capacity in wireless sensor and ad hoc networks. This definition is general and in the special case of random networks, it becomes equal to the bits per Joule definition of [16]. The formal definition is as follows.

DEFINITION 5.1. [Energy Efficiency: bit-meters per joule] In random wireless ad hoc networks with limited energy, the energy efficiency is

$$
\eta(n)=\frac{\lambda(n)}{P(n)}
$$

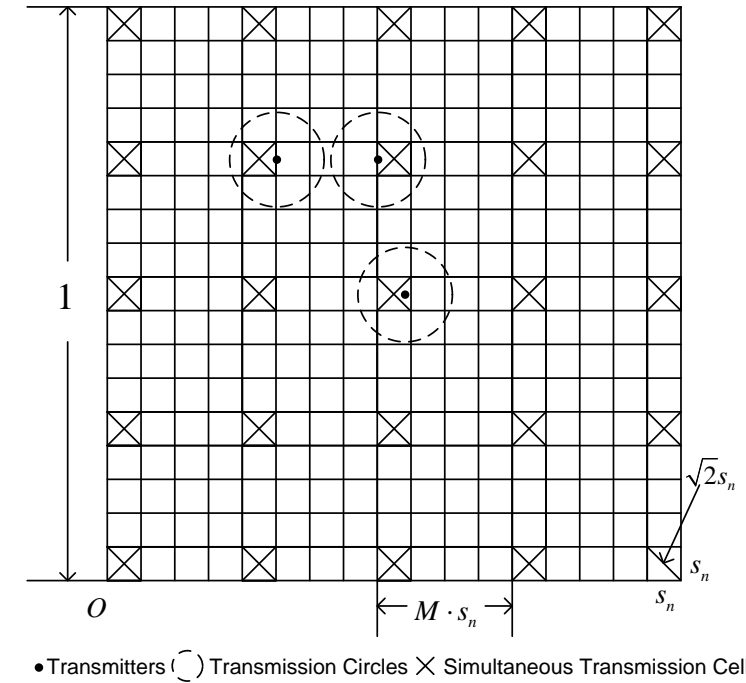

Figure 3: A tessellation of the unit square into small square cells. The shaded cells are concurrently transmitting cells with $M=4$

where $\lambda(n)$ is the transport capacity of the network and $P(n)$ is the total minimum average power required to achieve $\lambda(n)$ for each source-destination pair in the network.

With this definition of efficiency, we compute the relationship between transport capacity and the energy efficiency for the various approaches defined to increase the transport capacity of wireless ad hoc networks, including our own.

\subsection{Energy Efficiency in Approach by Gupta and Kumar [8]}

The lower bound for the transport capacity in [8] is derived by dividing the network area into $M^{2}$ disjoint groups as shown in Fig. 3 (crossed squares in this figure are one group) and at each time slot, only one group is allowed to communicate. Using a multi-hop routing protocol and assuming that at most one transmission is successful at a receiver, the transport capacity under the physical model is lower bounded as

$$
\lambda(n)=\Theta\left(\frac{1}{\sqrt{n \log n}}\right) \mathrm{bits} / \mathrm{sec} .
$$

The $S I N R$ at each receiver when only nodes in one group are allowed to transmit is computed as follows. Suppose each small square in this figure has a side length of $s_{n}=$ $\Theta\left(\sqrt{\frac{\log n}{n}}\right)$. The lower bound for SINR is achieved when we have the maximum distance between transmitter and the receiver node, i.e., $d=2 \sqrt{2} \Theta\left(\sqrt{\frac{\log n}{n}}\right)=2 \sqrt{2} s_{n}$, and the interfering nodes for each hop have the least distance, i.e., $(k M-1) s_{n}$ where $k$ is the hop distance from the receiver node. Thus, for any transmitter-receiver pair, the SINR is lower bounded as

$$
S I N R \geq \frac{P\left(2 \sqrt{2} s_{n}\right)^{-\alpha}}{B N_{0}+P \sum_{k=1}^{\infty} 8 k\left((k M-1) s_{n}\right)^{-\alpha}}
$$


It is easy to show that the minimum transmit power for each hop $P$ to guarantee that the $S I N R \geq \beta$ is

$$
\min (P)=\Theta\left(s_{n}^{\alpha}\right)=\Theta\left(\left(\frac{\log n}{n}\right)^{\frac{\alpha}{2}}\right) .
$$

The average number of hops from source to destination is $\frac{1}{s_{n}}=\Theta\left(\sqrt{\frac{n}{\log n}}\right)$. Therefore, the total average power to transmit information from any source to its destination is given by

$$
\begin{aligned}
P(n) & =\min (P) \times \text { total number of hops } \\
& =\Theta\left(\left(\frac{\log n}{n}\right)^{\frac{\alpha}{2}-\frac{1}{2}}\right) .
\end{aligned}
$$

The energy efficiency for this scheme can be computed by dividing the transport capacity by the total average power required to achieve this capacity. This renders

$$
\eta(n)=\Theta\left(\frac{n^{\frac{\alpha}{2}-1}}{(\log n)^{\frac{\alpha}{2}}}\right) .
$$

\subsection{Energy Efficiency in Approach by Franceschetti et al. [3]}

The communication in the approach by Franceschetti et al. [3] is based on dividing the transfer of packets into four phases. In the first phase, the source transmits a packet to a relay inside a path that is called "highway path." The distance between the source and highway path is considered a long range communication and is proportional to $\Theta\left(\frac{\log n}{\sqrt{n}}\right)$. Inside the highway path in phases two and three, multiple hop communication occurs horizontally and vertically respectively. The communication range is of short range and proportional to $\Theta\left(\frac{1}{\sqrt{n}}\right)$. Communication in phase four is similar to phase one and it is between relay and destination.

Assume that $P_{h}(n)$ is the transmit power at the highway path in phases two and three. Following the definition in [3], the interference from the other cells can be expressed as

$$
I(d, n) \leq P_{h}(n)\left(s_{n}(d+1)\right)^{-\alpha} \sum_{k=1}^{\infty} 8 k(2 k-1)^{-\alpha} .
$$

Because the summation in (28) converges to a constant value $c_{6}$, then the total interference is upper bounded as

$$
I(d, n) \leq P_{h}(n)\left(s_{n}(d+1)\right)^{-\alpha} c_{6} .
$$

The signal power at the receiver is lower bounded as

$$
S(d, n) \geq P_{h}(n)\left(s_{n} \sqrt{2}(d+1)\right)^{-\alpha} .
$$

Using the above results, the $S I N R$ is derived as

$$
\begin{aligned}
S I N R & =\frac{S(d, n)}{B N_{0}+I(d, n)} \\
& \geq \frac{P_{h}(n)(\sqrt{2})^{-\alpha}}{B N_{0}\left(s_{n}(d+1)\right)^{\alpha}+P_{h}(n) c_{6}} .
\end{aligned}
$$

In the limit, the minimum required power to guarantee that the $S I N R$ satisfies the physical model when $n \rightarrow \infty$ is

$$
\min \left(P_{h}(n)\right)=\Theta\left(\left(s_{n}(d+1)\right)^{\alpha}\right)=\Theta\left((n)^{-\alpha / 2}\right) .
$$

For the long-range communications in the first and fourth phase, there is no interference. Therefore, the SINR can be expressed as

$$
S I N R=\frac{P_{u}\left(\frac{\log n}{\sqrt{n}}\right)^{-\alpha}}{B N_{0}} .
$$

The minimum required power for this case to guarantee the physical model condition is given by

$$
\min \left(P_{u}\right)=\Theta\left(\left(\frac{(\log n)^{2}}{n}\right)^{\frac{\alpha}{2}}\right) .
$$

Using the definition of energy efficiency, we can compute its value for this case as

$$
\begin{aligned}
\eta(n) & =\lambda(n) / P(n)=\frac{\lambda(n)}{2 \min \left(P_{u}\right)+\sqrt{n} \min \left(P_{h}\right)} \\
& =\Theta\left(n^{\frac{\alpha}{2}-1}\right) .
\end{aligned}
$$

\subsection{Energy Efficiency in Approach by Ozgur et al. [14]}

Ozgur et al. [14] proposed a hierarchical cooperation method to achieve linear scalability with virtual MIMO techniques. In this method, the communication range is of order $\Theta(1)$ and, therefore, $P_{\min }=\Theta(1), \lambda(n)=\Theta(1)$, and $\eta(n)=\Theta(1)$. Note again that we do not consider the signaling overhead required for cooperation in this approach. We only consider the throughput capacity in the physical layer.

\subsection{Energy Efficiency with MPR}

In this paper, we demonstrated that MPR closes the gap between the upper and lower bounds of the capacity of wireless ad hoc networks by achieving higher transport capacity. However, it is important to find out the energy efficiency of this approach. From the derivation of transport capacity for MPR in (21), the SINR is given by

$$
S I N R \geq \frac{P(R(n))^{-\alpha}}{B N_{0}+\frac{\pi}{2} n R^{2}(n) \sum_{i=1}^{l_{\Gamma} / 2 D(n)} 2 P(i D(n)-R(n))^{-\alpha}}
$$

The physical model constraint is guaranteed for SINR asymptotically when the minimum transmit power $P_{\mathrm{MPR}}(n)$ is

$$
\min \left(P_{\mathrm{MPR}}(n)\right)=\Theta\left(R^{\alpha}(n)\right)=\left(\frac{\log n}{n}\right)^{\frac{\alpha}{2}} .
$$

Eq. (35) is derived using Eqs. (20) and (22) when $n \rightarrow \infty$.

The relationship between $\lambda(n)$ and $P_{\mathrm{MPR}}(n)$ can be computed from Theorem 4.6 as

$$
\lambda(n)=n^{-1 / \alpha}\left(P_{\mathrm{MPR}}(n)\right)^{\frac{\alpha-2}{\alpha^{2}}} .
$$

Because the communication range in MPR is equal to $R(n)$, the total minimum transmit power from source to destination is equal to $\frac{P_{\mathrm{MPR}}(n)}{R(n)}$.

The energy efficiency of MPR scheme is given by

$$
\begin{aligned}
\eta(n) & =\frac{\lambda(n) R(n)}{P_{\mathrm{MPR}}(n)}=\lambda(n)(R(n))^{1-\alpha} \\
& =n^{-\frac{\alpha-1}{\alpha-2}} \lambda(n)^{\frac{-(\alpha-1)^{2}-1}{\alpha-2}} .
\end{aligned}
$$

\section{DISCUSSION}

The reason for the significant increase in capacity with MPR is that, unlike point-to-point communication in which 


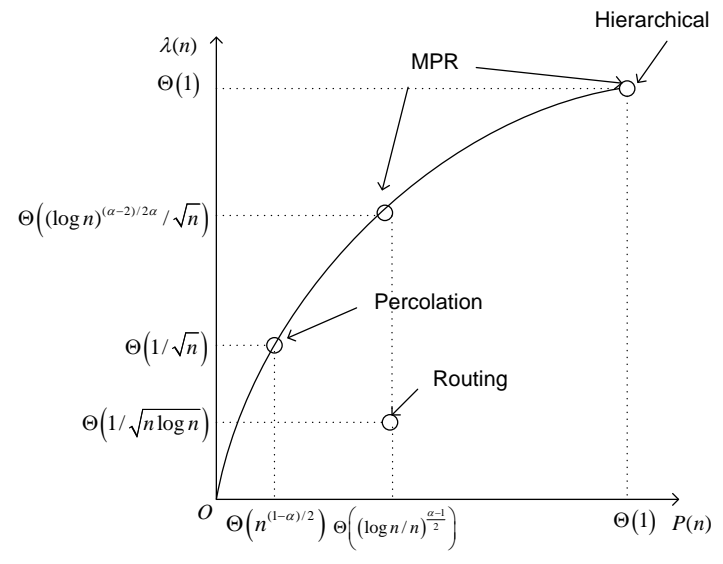

Figure 4: Power and Capacity tradeoff

nodes compete to access the channel, MPR embraces (strong) interference by utilizing higher decoding complexity for all nodes. It is interesting to note that recent work on network coding $[9,23]$ implicitly assume the ability of the nodes to receive multiple concurrent transmissions, i.e., some for of MPR. These results clearly demonstrate that embracing interference is crucial to improve the performance of wireless ad hoc networks, and that MPR constitutes an important component of that.

Another interesting observation is the fact that increasing the receiver range $R(n)$ increases the throughput capacity. This is in sharp contrast with point-to-point communication in which increasing the communication range actually decreases the throughput capacity and it is again due to the fact that MPR embraces the interference.

Figure 4 shows the tradeoff between the total minimum transmit power and the transport capacity. From this figure, it is clear that the total transmit power for the network must be increased in order to increase the per source-destination transport capacity in random wireless ad hoc networks.

Figure 5 shows that, by increasing the transport capacity in wireless ad hoc networks, the energy efficiency of all the schemes we analyzed decreases. Many wireless ad hoc networks are limited in total available energy or power for each node. Therefore, increasing the transport capacity may not be feasible if the required power to do so is not available. This result also shows that the transport capacity should not be the only metric used in evaluating and comparing the merits of different schemes. The energy efficiency of these schemes is also very important. Based on different values for $R(n)$, different transport capacities can be attained. In general, MPR allows to have tradeoff between receiver complexity and transport capacity.

There are certain issues that we did not discuss in this paper. Our analysis does not include the energy required for increased decoding complexity, which is necessary for MPR. Our analysis also does not include the additional required overhead related to cooperation among nodes. Such topics are the subject of future studies.

\section{CONCLUSION}

This paper shows that the use of MPR can close the gap for the transport (throughput) capacity in random wireless

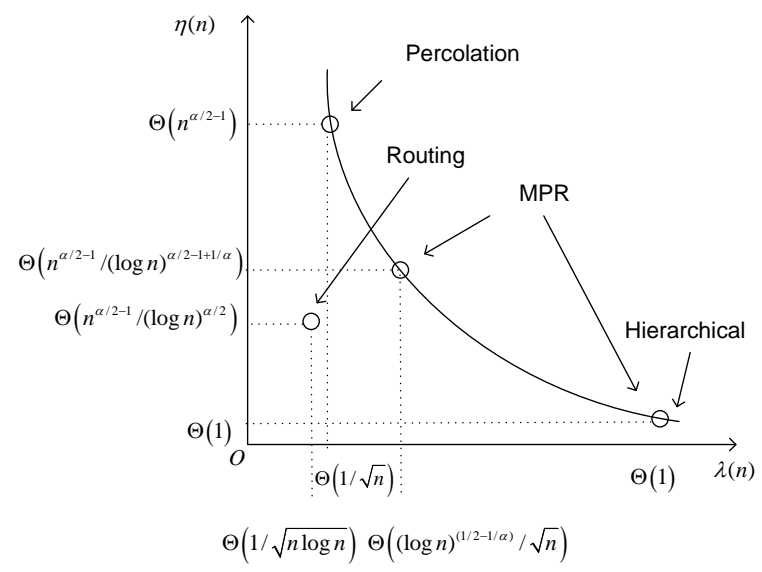

Figure 5: Capacity and energy efficiency tradeoff

ad hoc networks under the physical model, while achieving much higher capacity gain than that of [3]. The tight bound is $\Theta\left(\frac{(R(n))^{1-2 / \alpha}}{\left(n^{(1 / \alpha)}\right)}\right)$ where $R(n)$ is the receiver range in MPR model. For the minimum value of $R(n)$, a gain of $\Theta\left((\log n)^{\frac{\alpha-2}{2 \alpha}}\right)$ is achievable in MPR scheme.

We introduced a new definition related to energy efficiency based on bit-meters per Joule metric. Our results show that increasing the transport capacity by means of MPR or any of the other techniques proposed to date $[3,8,14]$ results in a reduction of energy efficiency in the network. Accordingly, there is a tradeoff to be made between increasing transport capacity and decreasing energy efficiency. Determining what is the optimum tradeoff between capacity and energy efficiency is an open problem.

\section{ACKNOWLEDGMENTS}

This work was partially sponsored by the U.S. Army Research Office (ARO) under grants W911NF-04-1-0224 and W911NF-05-1-0246, by the National Science Foundation (NSF) under grant CCF-0729230, by the Defense Advanced Research Projects Agency (DARPA) through Air Force Research Laboratory (AFRL) Contract FA8750-07-C-0169, and by the Baskin Chair of Computer Engineering. The views and conclusions contained in this document are those of the authors and should not be interpreted as representing the official policies, either expressed or implied, of the Defense Advanced Research Projects Agency or the U.S. Government.

\section{REFERENCES}

[1] de Moraes, R. M., Sadjadpour, H. R., And Garcia-LunA-Aceves, J. J. Many-to-many communication: A new approach for collaboration in manets. In Proc. of IEEE INFOCOM 2007

(Anchorage, Alaska, USA., May 6-12 2007).

[2] El Gamal, A., Mammen, J., Prabhakar, B., and SHAH, D. Optimal throughput-delay scaling in wireless networks part i: The fluid model. IEEE Transactions on Information Theory 52, 6 (2007), 2568-2592.

[3] Franceschetti, M., Dousse, O., Tse, D., and Thiran, P. Closing the gap in the capacity of wireless 
networks via percolation theory. IEEE Transactions on Information Theory 53, 3 (2007), 1009-1018.

[4] Garcia-Luna-Aceves, J. J., Sadjadpour, H. R., AND WANG, Z. Challenges: Towards truly scalable ad hoc networks. In Proc. of ACM MobiCom 2007 (Montreal, Quebec, Canada, September 9-14 2007).

[5] Ghez, S., Verdu, S., ANd Schwartz, S. Stability properties of slotted aloha with multipacket reception capability. IEEE Transactions on Automatic Control 33, 7 (1988), 640-649.

[6] Ghez, S., Verdu, S., And Schwartz, S. Optimal decentralized control in the random access multipacket channel. IEEE Transactions on Automatic Control 34, 11 (1989), 1153-1163.

[7] Grossglauser, M., And Tse, D. Mobility increases the capacity of ad hoc wireless networks. IEEE/ACM Transactions on Networking 10, 4 (2002), 477-486.

[8] Gupta, P., And Kumar, P. R. The capacity of wireless networks. IEEE Transactions on Information Theory 46, 2 (2000), 388-404.

[9] Katti, S., Gollakota, S., and Katabi, D. Embracing wireless interference: Analog network coding. In Proc. of ACM SIGCOMM 2007 (Kyoto, Japan, August 27-31 2007).

[10] Kyasanur, P., And Vaidya, N. Capacity of multi-channel wireless networks: Impact of number of channels and interfaces. In Proc. of ACM MobiCom 2005 (Cologne, Germany, August 28-September 2 2005).

[11] Liu, J., Goeckel, D., And Towsley, D. Bounds on the gain of network coding and broadcasting in wireless networks. In Proc. of IEEE INFOCOM 2007 (Anchorage, Alaska, USA., May 6-12 2007).

[12] Motwani, R., and Raghavan, P. Randomized Algorithms. Cambridge University Press, 1995.

[13] Negi, R., and Rajeswaran, A. Capacity of power constrained ad-hoc networks. In Proc. of IEEE INFOCOM 2004 (Hong Kong, March 7-11 2004).
[14] Ozgur, A., Leveque, O., And Tse, D. Hierarchical cooperation achieves optimal capacity scaling in ad hoc networks. IEEE Transactions on Information Theory 53, 10 (2007), 2549-3572.

[15] Peraki, C., And Servetto, S. On the maximum stable throughput problem in random networks with directional antennas. In Proc. of ACM MobiHoc 2003 (Annapolis, Maryland, USA., June 1-3 2003).

[16] Rodoplu, V., And Meng, T. Bits-per-joule capacity of energy-limited wireless networks. IEEE

Transactions on Wireless Communications 6, 3 (2007), 857-865.

[17] Tong, L., Zhao, Q., And Mergen, G. Multipacket reception in random access wireless networks: from signal processing to optimal medium access control. IEEE Communications Magazine 39, 11 (2001), 108-112.

[18] Toumpis, S., And Goldsmith, A. Capacity regions for wireless ad hoc networks. IEEE Transactions on Wireless Communications 2, 4 (2003), 736-748.

[19] Verdu, S. On channel capacity per unit cost. IEEE Transactions on Information Theory 36, 5 (1990), 1019-1030.

[20] Verdu, S. Multiuser Detection. Cambridge University Press, 1998

[21] Yi, S., Pei, Y., and Kalyanaraman, S. On the capacity improvement of ad hoc wireless networks using directional antennas. In Proc. of ACM MobiHoc 2003 (Annapolis, Maryland, USA. June 1-3 2003).

[22] Zhang, H., And Hou, J. Capacity of wireless ad-hoc networks under ultra wide band with power constraint. In Proc. of IEEE INFOCOM 2005 (Miami, FL, USA., March 1317 2005).

[23] Zhang, S., Liew, S., and Lam, P. Hot topic: Physical-layer network coding. In Proc. of ACM MobiCom 2006 (Los Angeles, California, USA., September 23-29 2006). 